\title{
Notas del manejo hemodinámico durante la guardia COVID-19
}

\section{Notes on hemodynamic management during the COVID-19 watch}

\author{
Dr. Enrique Monares-Zepeda, * Dr. Uriel Chavarría-Martínez, ${ }^{\ddagger}$ \\ Dr. Jesús Salvador Sánchez-Díaz ${ }^{\S}$
}

RESUMEN. Todo en medicina debe fundamentarse y equilibrarse en tres pilares. El primero es: un fuerte principio fisiológico; una explicación de qué provoca el fenómeno patológico al que nos estamos enfrentando y con la cual encontremos cómo puede ser revertida dicha patología. El segundo pilar es: una adecuada corroboración estadística; un principio fisiológico puede ser cierto, pero ello no implica que el desenlace que buscamos (disminución de la mortalidad) sea el resultado de nuestras intervenciones. El tercer pilar es un protocolo clínico, lo que implica la parte más importante de todas, el trabajar unidos. De nada sirve creer conocer la verdad, si es que es así, si no se tiene las mismas metas en todos los turnos, corremos el riesgo de caer en la falacia de que «lo que yo hago es lo correcto y los demás se equivocan»; si no estamos unidos en cada turno de atención al paciente, nunca sabremos qué es lo mejor para él, sólo tendremos un buen pretexto para afirmar que la culpa nunca es nuestra. Durante las crisis emergentes se puede trabajar sin la estadística mientras ésta se va construyendo, pero nunca sin fisiología y unidad (protocolos), la explicación fisiológica aquí vertida es lo más exacta posible, el protocolo es una inducción derivada de dicha fisiológica en espera de tener pronto una estadística que nos diga si lo que hacemos es de utilidad o no. En conclusión, lo que el lector tiene en sus manos son conjeturas en búsqueda de refutaciones. Al momento de escribir este artículo la única respuesta correcta es «Aún no lo sabemos».

ABSTRACT. Everything in medicine must be based and balanced on three pillars, the first is: a strong physiological principle; an explanation of what causes the pathological phenomenon that we are facing with which we find how this pathology can be reversed, the second pillar is an adequate statistical corroboration; a physiological principle may be true, but this does not imply that the outcome we seek (decrease in mortality) is the result of our interventions. The third pillar is a clinical protocol, which implies the most important part of all, working together. It is useless to believe that you know the truth, if that is the case, if you do not have the same goals in every shift, we run the risk of falling into the fallacy that «what I do is right and others are wrong», if we are not united in each shift of patient care we will never know what is best for the patient, we will only have a good pretext to affirm that the fault is never ours. During emerging crises you can work without statistics while it is being built, but never without physiology and unity (protocols), the physiological explanation given here is as accurate as possible, the protocol is an induction derived from said physiology, waiting to have Soon a statistic will tell us if what we do is useful or not. In conclusion, what the reader has in his hands are conjectures in search of refutations. At the time of writing this article the only correct answer is «We don't know yet».

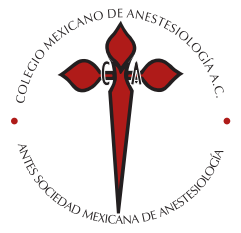

Palabras clave: COVID 19, monitoreo, hemodinamia, vasoconstricción pulmonar hipóxica, hipertensión pulmonar.

Keywords:

COVID 19, monitoring, hemodynamics, hypoxic pulmonary vasoconstriction, pulmonary hypertension.

* Médico Intensivista.

$\ddagger$ Pulmonary and Critical Care. Médico Intensivista, Neumólogo e Internista. Jefe Corporativo de Terapia Intensiva Hospitales Christus Muguerza, Programa de Trasplante Pulmonar Christus Muguerza, Programa de Rehabilitación Pulmonar del Hospital Universitario «Dr. José Eleuterio González».

$\S$ Médico Intensivista, Urgenciólogo. Unidad de Cuidados Intensivos

Adultos, Hospital de Especialidades Núm. 14 del Centro Médico Nacional «Adolfo Ruiz Cortines» del Instituto Mexicano del Seguro Social (IMSS). Veracruz, México.

Solicitud de sobretiros: Dr. Enrique Monares-Zepeda E-mail: enrique_monares @hotmail.com

Recibido para publicación: 04-04-2020

Aceptado para publicación: 08-04-2020

\section{MANEJO HEMODINÁMICO, CASOS CLÍNICOS DURANTE LA GUARDIA COVID-19}

H l primer llamado de enfermería. Masculino de 59 años, antecedente de hipertensión, COVID-19 +, infiltrados bilaterales. Requirió intubación hace 48 horas y ahora se encuentra hipotenso con TA $89 / 45 \mathrm{mmHg}$ y con 2 horas de oliguria, en estos momentos con una $\mathrm{SO}_{2}$ de $85 \%$ que minutos antes se encontraba en $94 \%$ con mismo $\mathrm{FiO}_{2}$ de $65 \%$ y PEEP de $12 \mathrm{cmH}_{2} \mathrm{O}$, la $\mathrm{SvcO}_{2} 55 \%$. El residente de guardia cuestiona la posibilidad de evaluar la variabilidad de la presión de pulso o una prueba de levantamiento de pies para decidir administrar un reto de volumen de $30 \mathrm{~mL} / \mathrm{kg}$ de peso.
El segundo llamado de enfermería es por un ingreso. Paciente masculino de 71 años con diagnóstico de COVID-19 + , lleva tres días con fiebre de $38^{\circ} \mathrm{C}$, disnea Borg 5 de 6 , dolor torácico. La PCR es de $180 \mathrm{mg} / \mathrm{dL}$ y la ferritina 780 $\mathrm{pg} / \mathrm{dL}$. TA 75/40, frecuencia cardíaca de 130 por minuto. Se ha solicitado la evaluación del equipo ECMO venoarterial.

En caso de hipotensión, recomendamos un reto de vasopresores con principio fisiológico: los pacientes COVID-19 pueden desarrollar síndrome de insuficiencia respiratoria aguda (SIRA), el cual tiene cuatro momentos $\operatorname{críticos}^{(1)}$ a nivel hemodinámico hasta donde sabemos. El primero de ellos es una vasoconstricción pulmonar hipóxica ${ }^{(2)}(\mathrm{VCPH})$, la cual 
puede ocasionar hipertensión pulmonar y, a su vez, disfunción del ventrículo derecho. Éstas dos últimas situaciones pueden hacer que el paciente sea muy poco tolerante a cargas de volumen intravascular, lo que se puede evidenciar fácilmente por ultrasonido al encontrar un ventrículo derecho del mismo tamaño o mayor que el ventrículo izquierdo, junto con una vena cava inferior dilatada y sin colapso ${ }^{(3)}$. El segundo tipo de crisis hemodinámica de estos pacientes es cuando se encuentran en la llamada «tormenta de citoquinas» ${ }^{(4)}$, la cual está caracterizada por niveles de interleucina 6 altos, proteína C reactiva $>100 \mathrm{mg} / \mathrm{dL}$, ferritina $>600 \mu \mathrm{g}$, y eventos sostenidos de fiebre $>38^{\circ} \mathrm{C}$, la cual puede causar un estado de sepsis, vasodilatación y/o depresión miocárdica, incluyendo ruptura de placas ateromatosas en coronarias que lleven a infarto agudo de miocardio. El tercero ${ }^{(5,6)}$ son crisis relacionadas con eventos trombóticos, como la tromboembolia pulmonar, que se ha asociado a niveles de dímero $\mathrm{D}$ alto al ingreso de estos pacientes. El cuarto y más grave evento de crisis hemodinámica de estos pacientes es un cuadro de choque cardiogénico, causado por una miocarditis fulminante viral ${ }^{(7)}$.

Por lo tanto, no recomendamos hacer ninguna prueba estática o dinámica de respuesta a volumen por dos motivos: en primer lugar, los pacientes con algún grado de hipertensión pulmonar difícilmente son respondedores a volumen. Segundo y más importante, todas las pruebas dinámicas y/o estáticas de respondedor a volumen carecen de validez fisiológica ${ }^{(8)}$ en escenarios de hipertensión pulmonar. De hecho, la variabilidad de la presión de pulso puede verse aumentada como dato de disfunción de ventrículo derecho y no como un dato de respuesta a volumen ${ }^{(9)}$.

Evaluación del paciente 1. Por lo anterior, se decide no administrar volumen al paciente e iniciar norepinefrina a 0.03 $\mu \mathrm{g} / \mathrm{kg} / \mathrm{min}$, con lo cual la presión mejora a $111 / 75 \mathrm{mmHg}$ y con ello sin realizar cambios en el ventilador la $\mathrm{SaO}_{2}$ mejora a $91 \%$, lo que es una evidencia indirecta de que el aumento de presión arterial sistémica ha redistribuido el flujo de la circulación pulmonar y mejora el trastorno $\mathrm{V} / \mathrm{Q}$, en especial las áreas de bajo $\mathrm{V} / \mathrm{Q}$.

Si el escenario no es una tormenta de citocinas, la meta es lograr balances negativos, para lo cual empleamos el protocolo FACTT simplificado ${ }^{(10)}$ de restricción de volumen y empleo de diurético.

\section{Protocolo FACTT simplificado ${ }^{(10)}$ :}

1. Descontinúe fluidos de mantenimiento.

2. Dilución de medicamentos en la menor cantidad compatible posible.

3. No limite los requerimientos de nutrición enteral.

4. Utilice vasopresores en caso de $\mathrm{PAM}<60 \mathrm{mmHg}$.

\begin{tabular}{lll} 
& \multicolumn{2}{c}{ PAM $>60 \mathrm{mmHg}$} \\
\cline { 2 - 3 } PVC en $\mathrm{mmHg}$ & $\begin{array}{l}\text { Diuresis }<0.5 \\
\mathrm{~mL} / \mathrm{kg} / \mathrm{h}\end{array}$ & $\begin{array}{l}\text { Diuresis }<0.5 \\
\mathrm{~mL} / \mathrm{kg} / \mathrm{h}\end{array}$ \\
\hline$>8$ & $\begin{array}{l}\text { Furosemida* } 3 \mathrm{mg} / \mathrm{h} \\
\text { en infusión revalorar } \\
\text { en } 1 \text { hora }\end{array}$ & $\begin{array}{l}\text { Furosemida** } 20 \mathrm{mg} \\
\text { revalorar en } 4 \text { horas }\end{array}$ \\
& $\begin{array}{l}\text { Ringer lactato } 15 \\
\text { mL/kg peso revalorar } \\
\text { en 1 hora }\end{array}$ & $\begin{array}{l}\text { Furosemida** } 20 \mathrm{mg} \\
\text { revalorar en } 4 \text { horas }\end{array}$ \\
& $\begin{array}{l}\text { Ringer lactato 15 } \\
\mathrm{mL} / \mathrm{kg} \text { peso revalorar } \\
\text { en 1 hora }\end{array}$ & Revalorar en 4 horas \\
\hline $44 \mathrm{mmHg}$ & & \\
\hline
\end{tabular}

* Si el paciente se encuentra recibiendo esa dosis o mayor, duplique la dosis hasta un máximo de $24 \mathrm{mg} / \mathrm{h}$.

** Si el paciente ha recibido esa dosis de bolo o mayor, duplique la dosis hasta un máximo de $160 \mathrm{mg}$.

No exceda la dosis total de $620 \mathrm{mg} /$ día de furosemida.

Ante creatinina sérica $\geq 3 \mathrm{mg} / \mathrm{dL}$ durante el protocolo, evalúe la necesidad de hemodiálisis.

- Recomendamos preferir reto de vasopresor antes que reto de volumen para revertir eventos de hipotensión.

- No recomendamos la realización de pruebas dinámicas o estáticas para evaluar respuesta a fluidos.

- Recomendamos el protocolo FACTT simplificado para el manejo de volumen en pacientes con ARDS por COVID-19 que no se encuentren en eventos de tormenta de citocinas.

Evaluación del paciente 2. Los pacientes COVID-19 en crisis de tormenta de citocinas pueden ser ubicados en el fenotipo inflamatorio ${ }^{(11)}$ de ARDS y se ha descrito que este grupo no se beneficia de restricción de volumen ${ }^{(12)}$. Por el tiempo de duración de la fiebre, la estimación del déficit de volumen se inicia con un bolo de solución Ringer lactato de $250 \mathrm{~mL}$, al mismo tiempo que norepinefrina. De manera empírica recomendamos reto de volumen, sólo mientras se tenga presión venosa central $<8 \mathrm{mmHg}$.

- El equipo de intubación se prepara para realizar laringoscopía directa. Algunas recomendaciones hemodinámicas.

Norepinefrina ${ }^{(13)}$ puede redistribuir el flujo pulmonar, lo que disminuiría la hipertensión pulmonar, que es una de las causas de hipotensión de estos pacientes, pero dosis altas de norepinefrina pueden, por el contrario, exacerbar la hipertensión pulmonar, por lo que recomendamos antes de usar dosis altas de norepinefrina agregar vasopresina, ${ }^{(14)}$ la cual tiene una menor repercusión en la presión arterial pulmonar. Por otro lado, desde el punto de vista hemodinámico, recomendamos el empleo de fármacos de inducción con mínimos efectos hemodinámicos, que pueden consultarse en otros escritos al respecto. 


\section{Tabla 1: Escala pronóstica de cor pulmonale en SIRA(15).}

\begin{tabular}{lc} 
Parámetro & Puntos \\
\hline Neumonía como causa de SIRA & 1 \\
Meseta-PEEP $>18 \mathrm{cmH}_{2} \mathrm{O}$ & 1 \\
$\mathrm{PaO} 2 /$ FiO2 $<150$ & 1 \\
$\mathrm{PaCO} 2>48 \mathrm{mmHg}$ & 1 \\
Total & $0-4$ \\
\hline
\end{tabular}

SIRA = síndrome de insuficiencia respiratoria aguda; PEEP = presión positiva al final de la expiración.

Evolución del paciente 2. Se realiza carga de volumen 250 $\mathrm{mL}$, se inician vasopresores, así como ventilación mecánica, el paciente presenta TA $90 / 77 \mathrm{mmHg}$, FC 110 por minuto, $\mathrm{SaO}_{2} 94 \%, \mathrm{SvcO}_{2} 60 \%$, lactato $8 \mathrm{mmol} / \mathrm{L}$.

Es importante en lo posible contar con una evaluación ecocardiográfica. El manejo hemodinámico de estos pacientes se determina adecuado si se reduce el grado de hipertensión pulmonar y/o disminuye el tamaño del ventrículo derecho como respuesta a cada intervención. La presencia de cor pulmonale (hipertensión pulmonar) puede sospecharse mediante una escala diseñada por el grupo de trabajo de Vieillard-Baron ${ }^{(15)}$ :

La presencia de $>2$ puntos en la escala de cor pulmonale (Tabla 1) debe advertir de la presencia de algún grado de hipertensión pulmonar, y éste es el grupo de mayor mortalidad en pacientes con SIRA. En estos pacientes debe mantenerse el equilibrio de $\mathrm{PaCO}_{2}<48 \mathrm{mmHg}$ junto con presión meseta
$<27 \mathrm{cmH}_{2} \mathrm{O}$ y una relación meseta-PEEP $<18 \mathrm{cmH}_{2} \mathrm{O}$. Las cuales deben considerarse como parte de la estrategia hemodinámica de protección del ventrículo derecho ${ }^{(16)}$.

\section{Sospecha de hipoperfusión}

Criterios clínicos:

- Escala de piel ${ }^{(17)}$ marmórea $>2$.

- Llenado capilar ${ }^{(18)}>4$ segundos en rodillas; $>2.5$ segundos en dedo índice.

- Eventos de hipotermia ${ }^{(17)} \mathrm{T}<36^{\circ} \mathrm{C}$.

- Índice de choque (frecuencia cardíaca/presión arterial sistólica $>1$ ).

- Frecuencia cardíaca $<60$ x minuto + presión arterial sistólica $<90 \mathrm{mmHg}$.

Criterios gasométricos de bajo gasto:

- Saturación venosa central de oxígeno ${ }^{(19)}\left(\mathrm{SvcO}_{2}\right)<65 \%$.

- $\mathrm{Gap}^{(20)}$ venoarterial de $\mathrm{CO}_{2}=$ presión venosa de $\mathrm{CO}_{2}$ $\left(\mathrm{PvCO}_{2}\right)$ - presión arterial de $\mathrm{CO}_{2} \mathrm{PaCO}_{2}>6 \mathrm{mmHg}$.

Ante un criterio clínico más un criterio gasométrico, o tres criterios clínicos o los dos gasométricos, la probabilidad de hipoperfusión es muy alta.

Ante estos casos recomendamos los algoritmos de las Figuras 1 y 2 como una guía de abordaje. En caso de que

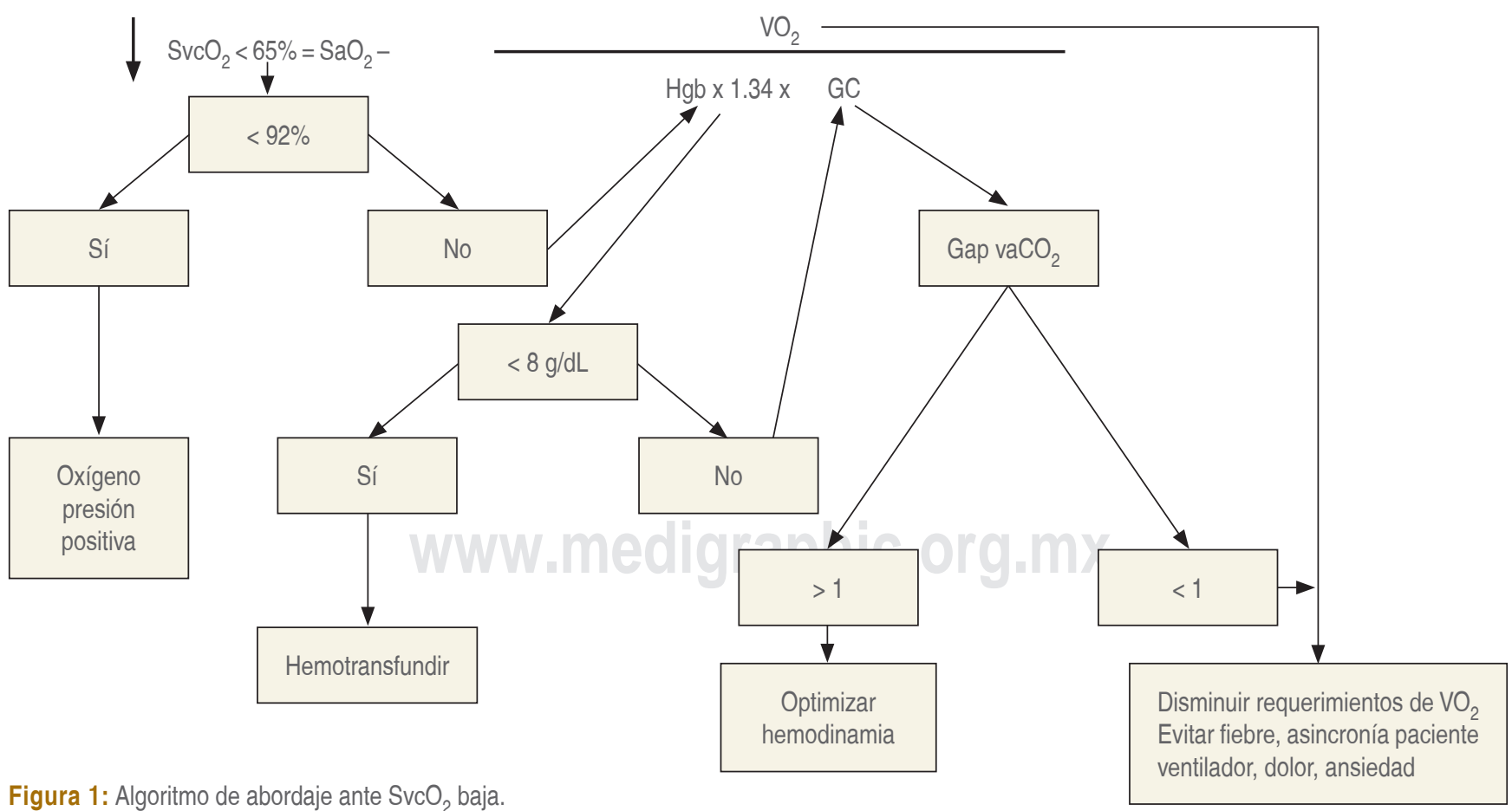




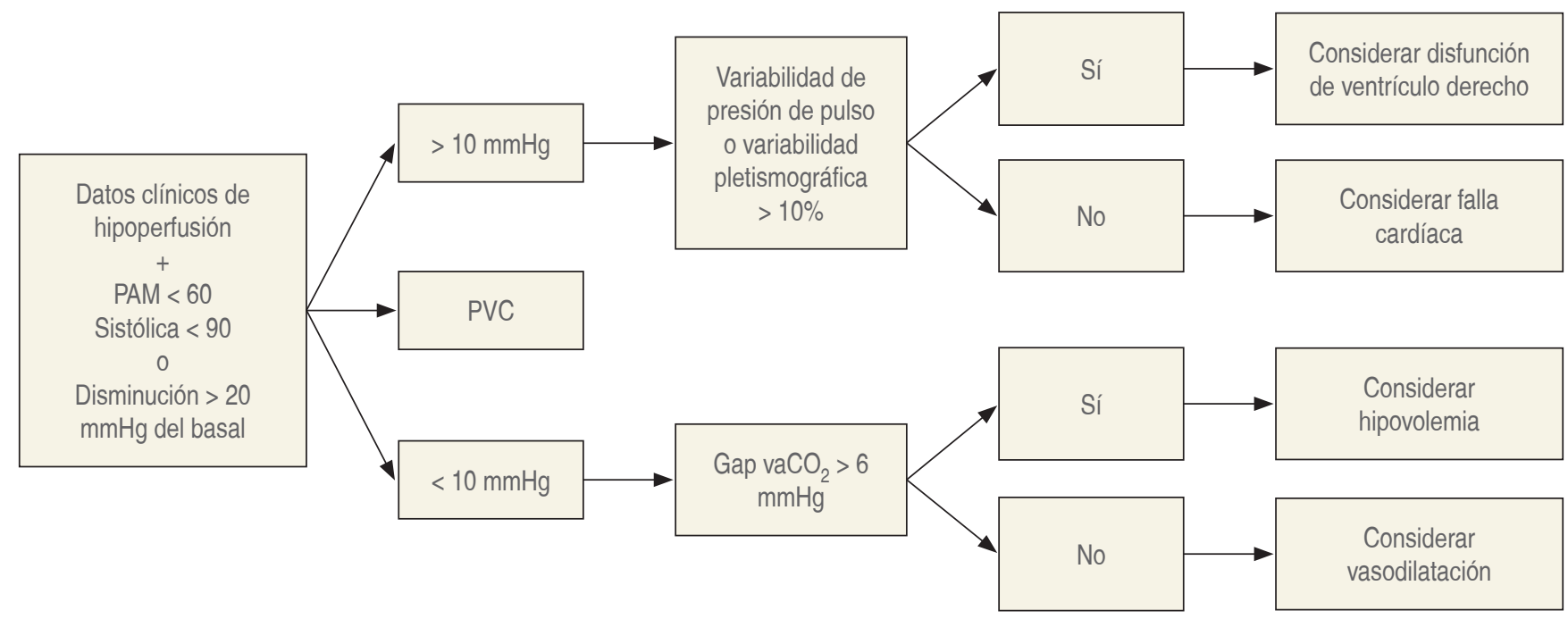

Figura 2: Algoritmo de abordaje ante hipotensión con datos de hipoperfusión.

el monitoreo ecocardiográfico o mínimamente invasivo no sea suficiente, y en especial en los casos donde se considere inodilatadores de arteria pulmonar, sugerimos monitoreo mediante catéter de arteria pulmonar.

Evolución paciente 2. El paciente continuó con deterioro hemodinámico, se inició dobutamina a ocho gammas, se reporta: $\mathrm{SvcO}_{2} 58 \%$, TA 90/45, FC 120 por minuto, lactato $12 \mathrm{mmol} / \mathrm{L}$. Se evalúa el inicio de ECMO VA por choque cardiogénico. Nosotros solicitamos la evaluación de un neumólogo con experiencia en hipertensión pulmonar para valorar fármacos de vasodilatación pulmonar.

\section{Recomendamos:}

- Evaluación ecocardiográfica siempre que sea posible.

- Ante eventos de tormenta de citocinas, recomendamos no seguir estrategias de restricción de líquidos, pero regresar a éstas una vez que se controle el evento.

- Evaluar el empleo de norepinefrina + vasopresina antes que dosis altas de norepinefrina en el manejo de hipotensión asociada con crisis de vasoconstricción pulmonar hipóxica.
- Un reto de inotrópico si continúa el deterioro hemodinámico con $\mathrm{SvcO}_{2}<60 \%$ y/o Gap vaCO${ }_{2}>6$ mmHg, así como seguimiento por parámetros de $\mathrm{SvcO}_{2}$ y Gap vaCO para evaluar respuesta terapéutica.

- Recomendamos en casos que persistan con deterioro hemodinámico atribuibles a hipertensión pulmonar la pronta consulta con expertos en hipertensión pulmonar, para evaluar terapias de vasodilatación pulmonar como óxido nítrico, iloprost, etcétera, y considerar el uso de monitoreo avanzado invasivo de la presión de la arteria pulmonar.

\section{CONCLUSIÓN}

Seguir protocolos bien estructurados puede ayudar a que rápidamente nos demos cuenta en dónde estamos equivocados, así como la dirección y magnitud de nuestro error. Por el momento, damos una base de lo que fisiológicamente hace sentido y esperamos pronto poder tener el pilar faltante de la estadística para respaldar este abordaje. Por lo pronto, si estas discusiones le ayudan a abordar mejor a los pacientes que están bajo su cargo, el trabajo a marchas forzadas empleado en redactarlas, habrá valido la pena con creces.

\section{REFERENCIAS}

1. Ruan Q, Yang K, Wang W, Jiang L, Song J. Clinical predictors of mortality due to COVID-19 based on an analysis of data of 150 patients from Wuhan, China [published online ahead of print, 2020 Mar 3]. Intensive Care Med. 2020; 1-3. doi: 10.1007/s00134-020-05991-x.

2. Ospina-Tascón GA, Bautista DF, Madriñán HJ, et al. Microcirculatory dysfunction and dead-space ventilation in early ARDS: a hypothesisgenerating observational study. Ann Intensive Care. 2020; 10 (1): 35. Published 2020 Mar 24. doi: 10.1186/s13613-020-00651-1.
3. Claure-Del Granado R, Mehta RL. Fluid overload in the ICU: evaluation and management. BMC Nephrol. 2016; 17: 109. Published 2016 Aug 2. doi: 10.1186/s12882-016-0323-6.

4. Mehta P, McAuley DF, Brown M, et al. COVID-19: consider cytokine storm syndromes and immunosuppression. Lancet. 2020; 395: 10331034. doi: 10.1016/S0140-6736(20)30628-0.

5. Zeng J, Huang J, Pan L. How to balance acute myocardial infarction and COVID-19: the protocols from Sichuan Provincial People's Hospital 
[published online ahead of print, 2020 Mar 11]. Intensive Care Med. 2020; 1-3. doi: 10.1007/s00134-020-05993-9.

6. Shi S, Qin M, Shen B, et al. Association of cardiac injury with mortality in hospitalized patients with COVID-19 in Wuhan, China [published online ahead of print, 2020 Mar 25]. JAMA Cardiol. 2020; e200950. doi: 10.1001/jamacardio.2020.0950.

7. Schunkert H, Erbel R. Evidenzbasierte Primärprävention: Wo stehen wir im Jahr 2020? [Evidence-based primary prevention: where do we stand in 2020?]. Herz. 2020; 45: 1-2. doi: 10.1007/s00059-019-04889-9.

8. Sondergaard S. Pavane for a pulse pressure variation defunct. Crit Care. 2013; 17: 327. Published 2013 Nov 14. doi: 10.1186/cc13109.

9. Vieillard-Baron A, Chergui K, Augarde R, et al. Cyclic changes in arterial pulse during respiratory support revisited by Doppler echocardiography. Am J Respir Crit Care Med. 2003; 168: 671-676. doi: 10.1164/ rccm.200301-135OC.

10. Grissom CK, Hirshberg EL, Dickerson JB, et al. Fluid management with a simplified conservative protocol for the acute respiratory distress syndrome*. Crit Care Med. 2015; 43: 288-295. doi: 10.1097/ CCM.000000000000715.

11. Wilson JG, Calfee CS. ARDS subphenotypes: understanding a heterogeneous syndrome. Crit Care. 2020; 24: 102. Published 2020 Mar 24. doi: 10.1186/s13054-020-2778-x.

12. Famous KR, Delucchi K, Ware LB, et al. Acute respiratory distress syndrome subphenotypes respond differently to randomized fluid management strategy. Am J Respir Crit Care Med. 2017; 195: 331-338. doi: 10.1164/rccm.201603-0645OC.

13. Tourneux P, Rakza T, Bouissou A, Krim G, Storme L. Pulmonary circulatory effects of norepinephrine in newborn infants with persistent pulmonary hypertension. J Pediatr. 2008; 153: 345-349. doi: 10.1016/j. jpeds.2008.03.007.

14. Mizota T, Fujiwara K, Hamada M, Matsukawa S, Segawa H. Effect of arginine vasopressin on systemic and pulmonary arterial pressure in a patient with pulmonary hypertension secondary to pulmonary emphysema: a case report. JA Clin Rep. 2017;3: 1. doi: 10.1186/s40981016-0072-3.

15. Mekontso Dessap A, Boissier F, Charron C, et al. Acute cor pulmonale during protective ventilation for acute respiratory distress syndrome: prevalence, predictors, and clinical impact. Intensive Care Med. 2016; 42: 862-870. doi: 10.1007/s00134-015-4141-2.

16. Repessé X, Vieillard-Baron A. Right heart function during acute respiratory distress syndrome. Ann Transl Med. 2017; 5: 295. doi: 10.21037/atm.2017.06.66.

17. Ferraris A, Bouisse C, Mottard N, et al. Mottling score and skin temperature in septic shock: Relation and impact on prognosis in ICU. PLoS One. 2018; 13: e0202329. Published 2018 Aug 16. doi: 10.1371/ journal.pone.0202329.

18. Pickard A, Karlen W, Ansermino JM. Capillary refill time: is it still a useful clinical sign? Anesth Analg. 2011; 113: 120-123. doi: 10.1213/ ANE.0b013e31821569f9.

19. Berridge JC. Influence of cardiac output on the correlation between mixed venous and central venous oxygen saturation. Br J Anaesth. 1992; 69: 409-410. doi: 10.1093/bja/69.4.409.

20. Mallat J, Lemyze M, Tronchon L, Vallet B, Thevenin D. Use of venousto-arterial carbon dioxide tension difference to guide resuscitation therapy in septic shock. World J Crit Care Med. 2016; 5: 47-56. Published 2016 Feb 4. doi: 10.5492/wjccm.v5.i1.47. 